\title{
Planning for smallpox outbreaks
}

\author{
Neil M. Ferguson ${ }^{1}$, Matt J. Keeling ${ }^{2}$, W. John Edmunds ${ }^{3}$, Raymond Gani ${ }^{4}$, Bryan T. Grenfell ${ }^{5}$, Roy M. Anderson ${ }^{1}$ \& Steve Leach ${ }^{4}$ \\ ${ }^{1}$ Department of Infectious Disease Epidemiology, Faculty of Medicine, Imperial College London, St Mary's Campus, Norfolk Place, London, W2 1PG, UK \\ ${ }^{2}$ Department of Biological Sciences and Mathematics Institute, University of Warwick, Gibbet Hill Road, Coventry, CV4 7AL, UK \\ ${ }^{3}$ Health Protection Agency, CDSC, 61 Colindale Avenue, London, NW9 5EQ, UK \\ ${ }^{4}$ Health Protection Agency, CAMR, Porton Down, Salisbury, Wiltshire SP4 0JG, UK \\ ${ }^{5}$ Department of Zoology, University of Cambridge, Downing Street, Cambridge, CB2 3EJ, UK
}

Mathematical models of viral transmission and control are important tools for assessing the threat posed by deliberate release of the smallpox virus and the best means of containing an outbreak. Models must balance biological realism against limitations of knowledge, and uncertainties need to be accurately communicated to policy-makers. Smallpox poses the particular challenge that key biological, social and spatial factors affecting disease spread in contemporary populations must be elucidated largely from historical studies undertaken before disease eradication in 1979. We review the use of models in smallpox planning within the broader epidemiological context set by recent outbreaks of both novel and re-emerging pathogens.

vents of recent years have heightened awareness of the potential threat of bioterrorism ${ }^{1}$, with smallpox considered to pose the greatest risk owing to the lethality (around 30\%, depending on age and other factors ${ }^{2,3}$ ) and transmissibility of the virus. Although once endemic in many human populations, smallpox was eradicated in 1979 largely as a result of mass vaccination reinforced by other highly focused control measures ${ }^{3}$. Today, although viral samples are officially retained in only two locations, the existence of other sources cannot be ruled out ${ }^{4}$. In the face of such a difficult to quantify, unlikely, but potentially serious threat, contingency planning demands a rational assessment of the scale of casualties that a smallpox attack might cause, and identification of what controls might be optimal in minimizing its effects ${ }^{5-8}$. The latter task is complicated by the severe adverse effects of vaccination experienced by a significant minority of individuals ${ }^{9,10}$, such that a national mass vaccination campaign could cause more deaths than an isolated smallpox epidemic. A second key problem is the passage of time since the last smallpox outbreak: human populations, mobility and patterns of social interactions have changed in the last 30 years, complicating extrapolation from historical epidemics to the prediction of future outbreaks.

Mathematical models of the transmission of infectious agents are valuable tools in making such assessments, because they can integrate epidemiological and biological data to give quantitative insights into patterns of disease spread and the effect of interventions. Examples include the design and evaluation of childhood disease immunization programmes ${ }^{11,12}$, predicting the demographic impact of the HIV epidemic in different regions ${ }^{13}$, and analysing the spread and control of the 2001 foot-and-mouth epidemic in Britain $^{14-17}$.

Following this philosophy, four recently published analyses of the potential spread of smallpox virus in modern urban communities all rely heavily on mathematical modelling ${ }^{5-8}$. Given that a key aim of these studies was to inform public health planning, it is unfortunate that their conclusions differ as to which type of vaccination strategy might be optimal, and on the scale of casualties likely to result from a smallpox attack. As mathematical modelling is the only way that we can examine the possible impact of different release and control scenarios, it is important to understand the strengths and weaknesses of different modelling approaches and how model assumptions affect the conclusions drawn.

\section{Epidemic dynamics}

At its simplest, an epidemic is a chain reaction of disease spread within a population (Box 1). The growth of an epidemic is principally governed by two factors: the number of secondary cases generated by one primary case at the start of the epidemic, termed the basic reproduction number or $R_{0}$, and the average time taken for the secondary cases to be infected by a primary case, termed the generation time or $T_{\mathrm{G}}$ (ref. 18). $R_{0}$ essentially determines how intensive a policy will need to be to control the epidemic, whereas both $T_{\mathrm{G}}$ and $R_{0}$ determine the time available to implement suitably intensive controls.

Thus, control policies for diseases which are highly infectious with short incubation periods, such as measles $\left(R_{0} \approx 17, T_{\mathrm{G}} \approx 11\right.$ days), tend to focus on the long-term reduction of the recruitment of susceptible people, through widespread childhood immunization $^{11,12,19}$. In contrast, smallpox is both less infectious $\left(R_{0}=4-10\right.$; refs 20,21$)$ and has a much longer incubation period $\left(T_{\mathrm{G}} \approx 21\right.$ days); thus, if an outbreak is detected in its earliest stages, there is sufficient time for localized control measures to be adopted. The feasibility of localized outbreak control during the final phase of the global smallpox vaccination programme, once a degree of 'herd immunity' had been created, is a key reason why smallpox was the first major viral pathogen to be eradicated worldwide ${ }^{3,6}$.

Evaluating the effectiveness of a potential control policy is not straightforward. A range of key epidemiological and social processes can have significant effects on the likely success of any given intervention. Greater model realism in describing disease biology and human contact patterns is needed to understand which controls work best under different conditions.

\section{Increasing model realism}

The simplest description of how the above processes and parameters govern disease transmission is represented mathematically by a crude model $^{18}$ (Box 1), which splits the population into 'susceptible', 'infected' and 'recovered' categories. Given its simplicity, this SIR model has been remarkable for the qualitative insights it has given into the epidemiology of a wide range of pathogens ${ }^{18,22}$. However, quantitative predictions that can be used in policy formulation, cost-benefit analysis or risk assessment frequently necessitate refinement of the basic model (Box 2), to allow for the inclusion of appropriate biological and behavioural factors.

Fundamental to the SIR model are assumptions that all susceptible people in the population are equally at risk of infection from any infected individual (that is, homogeneous mixing), and that all infected individuals have a constant and equal infectiousness. The latter assumption is clearly invalid for smallpox, where an uninfectious incubation period of about 12 days is typically followed by a 2-4-day prodromal period associated with mild symptoms and low infectiousness, then a highly infectious symptomatic period of 
about 9 days (Box 2). Inclusion of these disease stages within models of smallpox is essential if the effect of vaccination (or other control measures, such as quarantine) on infectiousness or mortality is to be represented realistically ${ }^{5-8}$.

Assuming homogeneous mixing of the population is also unrealistic-individuals tend to make contact with household members, workplace colleagues and friends at a much higher rate than random strangers, and such regular contacts will also tend to be in the same geographic vicinity. Hence socio-spatial structure has an important impact on transmission dynamics ${ }^{23}$ (see Box 2), particularly for diseases such as smallpox where close contact (for example,

\section{Box 1}

\section{Epidemics: basic theory}

Individuals affected by an epidemic move through a number of infection states:
S (susceptible)

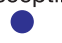

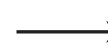

I (infected)

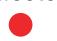

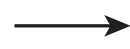

R (recovered)

Epidemics are spread through contact (for example, person to person):

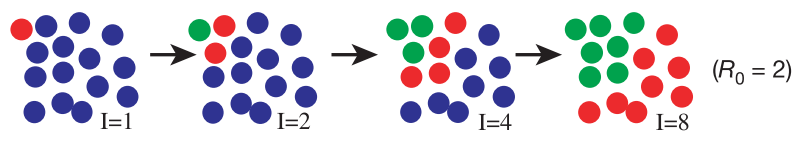

The 'chain reaction' nature of the process leads to exponential growth (once infected numbers are great enough to make random effects small), until the epidemic begins to run out of people to infect. This effect is summarized by the effective reproduction number $R$ (ref. 11), which declines from its maximum, $R_{0}$, as the susceptibles are depleted. Together, these processes give rise to the classic epidemic profile:

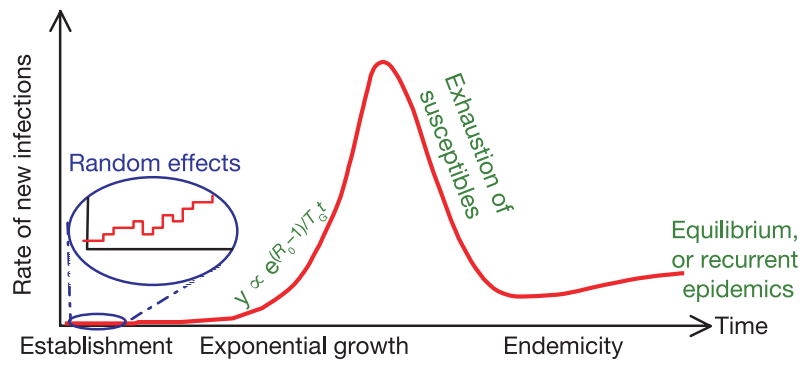

Controls either reduce susceptible numbers (such as vaccination) or limit transmission (for example, through movement controls). Both have the effect of reducing $R$ and slowing the spread of an epidemic; reducing $R$ below 1 means that the chains of transmission cannot be sustained and the epidemic dies out:

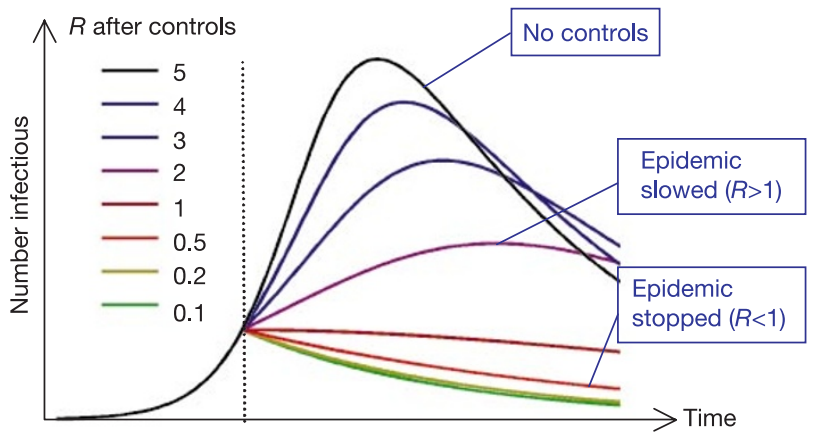

of the type more likely to occur in households and hospitals ${ }^{3}$ ) is usually required for transmission. However, incorporation of such detail into models considerably increases their complexity and the number of model parameters ${ }^{7}$ that need to be estimated.

\section{Assessing effectiveness of control strategies}

A variety of methods exist for controlling the spread of smallpox, ranging from different vaccination strategies to movement/contact restrictions placed on infectious cases and their contacts (Table 1). Thus a key aspect of policy-orientated epidemic modelling is to assess both the adequacy of current policy and how it might further be optimized. Optimality is principally the minimization of mortality and morbidity, so it is critical that models accurately incorporate expected adverse event rates from vaccination. However, the SARS virus has shown that the economic costs of an epidemic can be out of all proportion to the numbers infected, indicating that minimizing the duration of a smallpox outbreak might also be a critical priority when formulating control strategy.

In all cases, it is critical that models explicitly capture the underlying mechanism of the control policy being investigated. The net effect of a control policy on disease transmission is well characterized by its impact on $R$ : the effective reproduction number during the epidemic (Box 1). However, estimating this effect on $R$ without explicit modelling of the details of a control policy is practically impossible for complex models that include population heterogeneity and a realistic description of disease biology. Models which just assume a priori the effect of a control measure on $R$ (see for example refs 5,8 ) have little inferential power because the predicted effectiveness is determined by the assumed value of $R$.

In addition to $R$, other factors determine the effectiveness and likely success of any given control measure. These include the likely scale and geographic extent of any bioterrorist attack, associated risks or fatalities due to the control, the disruption to civic society, the level of vaccine uptake, overall resource requirements, and the ability of health agencies to implement policies. Models should therefore incorporate realistic logistical constraints on policy implementation $^{6}$, and, if needed, economic costs. There may also be a subtle interplay with epidemic dynamics here. For example, ring vaccination is constrained to the speed of the epidemic, whereas mass vaccination could in principle proceed as quickly as logistics allowed, thereby minimizing the duration of an outbreak.

\section{Model-based risk assessment}

A key benefit of using models to examine disease control options is their ability to explain and-with appropriate caveats-predict trends at a population level from interactions and processes at the individual level. Often the emergent dynamics at the population scale may be anything but obvious, owing to the many nonlinearities, complexities and feedbacks arising from the basic mechanisms at the individual level.

A corollary of the need to capture the mechanisms of transmission and control policies is that models have to be appropriately designed for the questions being addressed (Box 2). For instance, the feasibility of implementing different control policies is a key issue that will depend on the incidence of the disease. Models need to incorporate logistical constraints on policy implementation (for example, how many people can be vaccinated per $\mathrm{day}^{6}$ ) that are commensurate with that level of incidence. If the speed/risk of spread between communities is of interest, then models need to incorporate spatial structure. If contact tracing is to be assessed then some concept of social structure must be included.

Table 2 compares model structures and assumptions of four recent studies ${ }^{5-8}$. The diversity of model structures chosen and assumptions made regarding infection seeding, spread and control complicates direct comparison of the results of these studies. One issue for all the studies is the numbers of parameter values that are assumed, rather than estimated from data ${ }^{21}$. The most important 
factors determining the conclusions drawn by two of the studies ${ }^{6,7}$ are the assumed infectiousness during prodrome relative to that during symptomatic disease, and the relatively low effectiveness of isolation of symptomatic cases assumed. New best estimates from a well-observed historical epidemic in Africa indicate prodromal transmission alone contributes 0.16 to $R_{0}$ (ref. 21 ), $2.4 \%$ of the total $R_{0}$ value of 6.9. Both of the studies assumed that prodromal $R_{0}$ is $2.5-3$ out of a total $R_{0}$ of around 3 (refs 6,7 ). Analyses using the new estimates show that isolation and ring vaccination-if logistically feasible-is nearly always optimal, and never markedly worse than mass vaccination at minimizing mortality ${ }^{24}$.

The remaining two studies make simplifying assumptions that limit their usefulness. Reference 5 assumes purely exponential epidemic growth, meaning that little can be inferred about outbreaks of large size or duration. References 5 and 8 assume (rather than model) the impact of different control measures on transmission, meaning that their conclusions regarding control policy effectiveness are largely predetermined.

\section{Data and uncertainty}

The need to increase model sophistication and accuracy gives rise to an intrinsic tension: as model realism is increased the transparency associated with simple frameworks is often lost and the validation of model conclusions becomes harder. For models to be useful tools in policy planning, it is essential that they can be parameterized from available data, and tested against past and current epidemic outbreaks, with proper consideration of changes in human populations with respect to immunity, mobility and patterns of social interaction.

Simple models have fewer parameters, which tends to make parameter estimation easier. Conversely, more complex models may have dozens of parameters describing the details of disease biology, host movement patterns and population structure. Unless all these parameters are robustly estimated or the effect of uncertainty in their values explored, there is a danger that incorrect assumptions will be made (and obscured by the complexity); this can make more detailed models no more reliable (and sometimes less so) than simpler frameworks. Achieving the correct balance between model complexity and validation is therefore key to informative modelling (Box 2 and Table 2). Validation in this context is how well the model matches observed epidemic behaviour at the level of detail relevant to the model's purposes. Ideally, such data should be independent of any epidemiological data used to estimate model parameters. When this ideal is not achievable (for example, when analysing an emerging epidemic of a novel disease $^{25,26}$ ), the use of rigorous statistical methods for assessing model goodness of fit is imperative.

The most useful data for parameterization and validation of models are detailed (individual case) reports from historical outbreaks ${ }^{2,3}$. Such data provide the only reliable estimates of the reproduction number of smallpox (4-10, depending on the out-

Box 2

Modelling complexities

\section{Greater disease realism}

Extend the SIR model to incorporate the known within-host disease behaviour:

\begin{tabular}{|l|l|l|c|}
\hline $\begin{array}{l}\text { Latent/incubation } \\
\text { period }\end{array}$ & $\begin{array}{l}\text { Prodromal } \\
\text { period }\end{array}$ & $\begin{array}{l}\text { Symptomatic/ } \\
\text { infectious period }\end{array}$ & Recovery period \\
\hline Duration: 12 days $( \pm 3) \quad 3$ days $( \pm 2) \quad 9$ days $( \pm 5)$ & 17 days $( \pm 9)$ \\
\hline
\end{tabular}

Relative infectiousness in the prodromal and symptomatic periods is crucial in determining the optimal control strategy, with greater prodromal spread favouring mass vaccination.

\section{Capturing social/spatial structure}

Homogeneous mixing. Standard assumption of simple models-an individual has an equal chance of contacting anyone in the population. Contacts are independent (individuals who have previously made contact have no more or less chance of contacting again).

Age/social structure. Individuals have different probabilities of contact within specified population subgroups and between them. Subgroups might be defined by age, occupation (for example, hospital, school), socio-economic or health factors.
Network structure. Individuals form stable contact networks (for example, household, work colleagues, friends), the structure of which determines transmission dynamics. There can be rapid localized spread, followed by slow down as depletion of local susceptibles occurs due to correlations and clustering.

Patch structure. Populations are clumped, so towns/cities are natural units to study. Thus 'patch' (metapopulation) models are a valuable tool. There is more mixing within a patch than between patches, and patches can be out of sync in terms of epidemic progression.

Individually based models. Stochastic simulation of contact patterns and disease progression at the level of individuals allows models to capture arbitrary levels of heterogeneity (including network and geographic) structure. However, such models are both computationally and data intensive if rigorous validation and parameter estimation is to be performed.

\section{Stochastic or deterministic models?}

Deterministic (clockwork) models are rapid to simulate, relatively easy to parameterize, and hopefully capture the average epidemic behaviour. Stochastic models recognize the random nature of transmission events. As such they allow an assessment of the variability of the epidemic behaviour and are essential to deal with the low levels of infection near the start and end of an epidemic ${ }^{30}$.

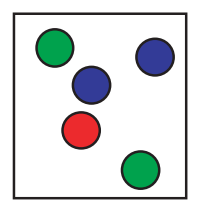

Homogenous mixing

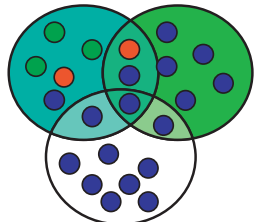

Age/social structure

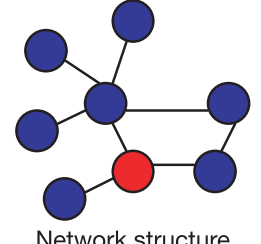

Network structure

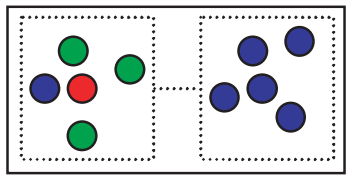

Patch structure

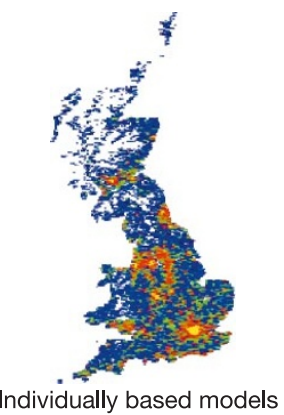




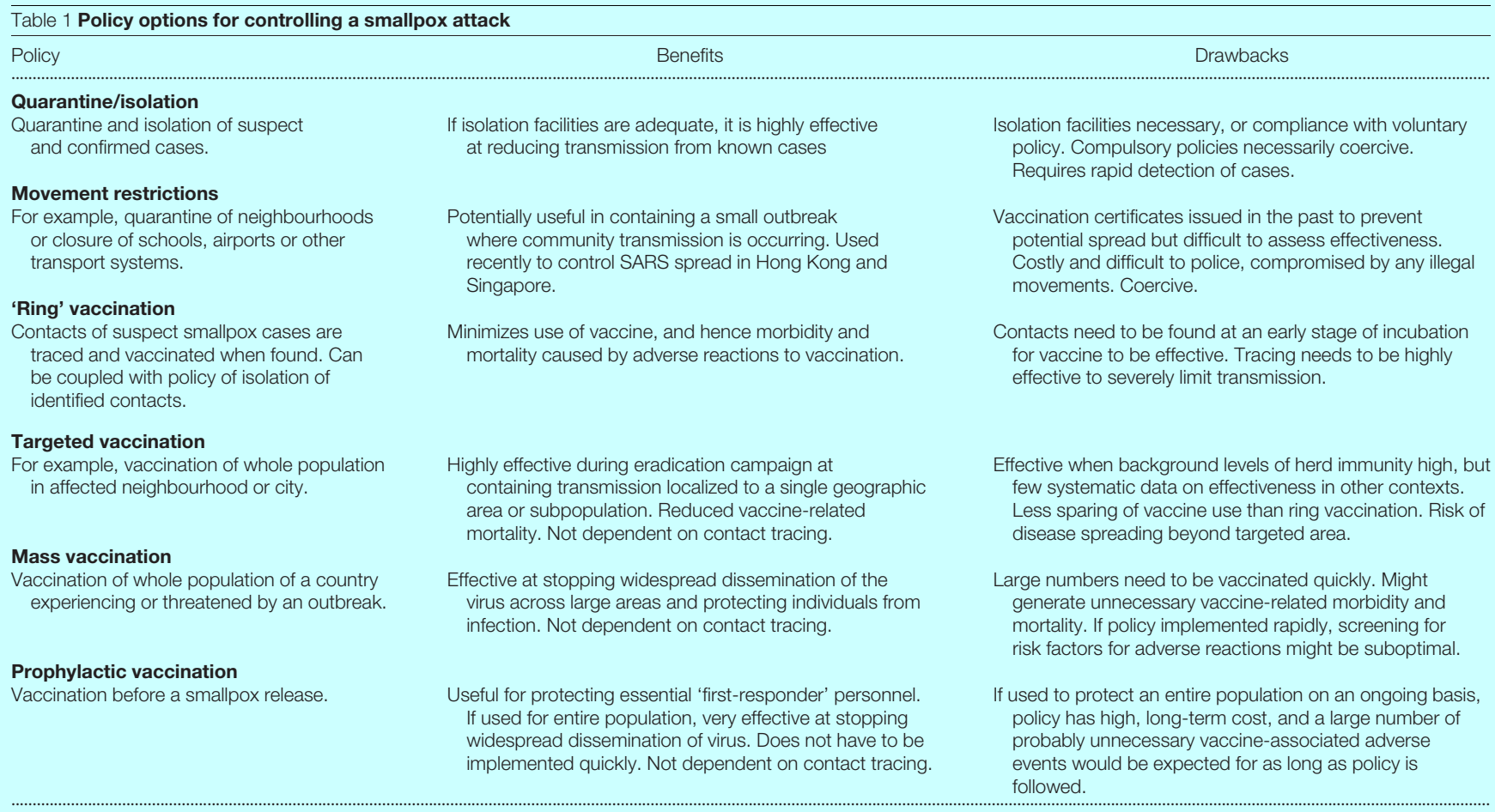

These policy options are unlikely to be applied in isolation of each other and will necessarily depend on availability of resources and levels of preparedness.

break $^{20,21}$, and information on how infectiousness varies at different disease stages ${ }^{2,21}$. However, extrapolating from historical data to contemporary developed-world populations is problematic. It is unclear how much residual immunity remains today as a result of past vaccination programmes; those vaccinated 25-30 years ago are unlikely to possess complete immunity and a significant proportion may develop less severe forms of the disease ${ }^{2,3}$, potentially changing the dynamics of transmission. Allowing for past immunity levels is therefore critical when estimating $R_{0}$ from historical data ${ }^{20}$. Historically, most infections occurred in care-givers to symptomatic individuals, whether in households or hospitals ${ }^{3}$. It is unclear how
30 years of changes in household sizes, working patterns and mobility would affect transmission patterns today (see Box 2). Incorporating detailed data on demographics and human mobility into spatially explicit models offers one method by which such extrapolation can be made more reliable, but the scale of changes mean that much uncertainty will inevitably remain.

With such uncertainty, it is critical that risk assessment studies use modern statistical methods ${ }^{27}$ to obtain the best possible parameter estimates from historical data, while allowing for changes in the last 30 years. However, historical data are less relevant for some key parameters-such as the likely scale of a bioterrorist attack, how

Table 2 Summary of recent smallpox modelling studies

Study Key features

Meltzer et al. (ref. 5)

(1) Homogeneous mixing; (2) stochastic; (3) no social/spatia structure; (4) $R_{0}=1.5-3$; (5) vaccination not directly modelled but in conjunction with quarantine is assumed to reduce $R$ to below 1 ; (6) no depletion of susceptibles; (7) 100 people initially infected

Kaplan et al. (ref. 6)

(1) Homogeneous mixing in large population (10 million); (2) deterministic; (3) no social/spatial structure; (4) $R_{0}=3$ for base case (range 1-20); (5) mass vaccination and ring vaccination compared; (6) considers public health logistical constraints; (7) only those 'asymptomatic' (that is in prodrome) are infectious - those symptomatic (with rash) are assumed to be isolated; (8) number initially infected $=1-100,000$

Halloran et al. (ref. 7)

(1) Heterogeneous mixing (social structure included) in small population (2,000); (2) stochastic; (3) $R_{0}=3.2$; (4) mass vaccination and ring vaccination compared; (5) considers current residual herd immunity; (6) number initially infected $=1-10$

Bozzette et al. (ref. 8) $\quad$ (1) Homogeneous mixing; (2) stochastic; (3) $R$ (no control) $=15$ 3.4 and 1.8 in hospital, mixed and community outbreaks respectively; $\left(R_{0} \geq R\right.$ (no control)); (4) compares mass vaccination, ring vaccination and prophylactic vaccination of healthcare workers; (5) considers vaccine-related adverse events; (6) number initially infected $=2-100,000$

\section{Comments}

(1) Estimates required control effort by correlating vaccine doses used against number of cases in historical outbreaks; (2) number of doses is not related to the number of cases, but to the level of susceptibility (and $R_{0}$ ); (3) model substantially overestimates cases because depletion of susceptibles is not modelled; (4) controls assumed to reduce $R$ to 0.99 , that is, programme effectiveness is model input

(1) $R_{0}$ assumed derived from historical estimates, but level of prodromal transmission assumed much greater than new best estimates derived from historical data ${ }^{21}$; (2) assumption that transmission occurs during the prodromal period biases results in favour of mass vaccination; (3) assumption of homogeneous mixing will also bias the results in favour of mass vaccination; (4) considers vaccine-related deaths only in contraindicated people, and not those in non-contraindicated individuals or other adverse events

(1) Large number of parameters assumed, particularly regarding mixing patterns, limited sensitivity analysis performed; (2) relatively large proportion (0.05-0.5\%) of community initially infected; (3) model of small community of 2,000 individuals; (4) assumption that $>75 \%$ of transmission occurs during prodrome biases results in favour of mass vaccination

(1) Assumes the effect of the control policies on $R$, based on review and 'judgement'; that is, effectiveness of policies is an input into the model rather than an output; the model therefore has little explanatory or predictive power; (2) large number of parameters assumed, limited sensitivity analysis performed; (3) assumes disease has no effect on the depletion of susceptibles; (4) assessment of threat of attack is subjective 
rapidly the disease would be recognized, and the ability of public health authorities to respond. Furthermore, it is unclear how population behaviour would change in the face of an epidemic. Before recognition of the outbreak, would individuals in the latter stages of prodrome have more or fewer contacts, compared with their historical counterparts? Once smallpox is identified, will people voluntarily restrict their movements, or attempt to flee urban centres? These factors need to be explored with robust analysis $^{28,29}$ of the sensitivity of model results and predicted optimal controls to parameter assumptions.

Particularly important is the assessment of the potential for catastrophic outcomes. A policy option may be optimal for the great majority of possible parameter scenarios, but fail to control disease spread in a few worst-case scenarios, which are still feasible given current data. An alternative policy option that is slightly less optimal for most scenarios but controls spread in the worst case might then be preferred.

For models to have a meaningful role in influencing policy decisions, it is therefore critical that not just 'most likely' or 'worst case' scenario modelling results are communicated but that a more detailed understanding of the sensitivity of predictions of outbreak size and policy optimality to model assumptions is conveyed, together with open acknowledgement of model or data weaknesses. Once an outbreak has begun, such questions may also be answered more precisely through the use of real-time modelling to refine parameters and better inform policy.

\section{Conclusions}

Given the many uncertainties outlined above, we argue that no model can be truly predictive in the context of smallpox outbreak planning, and no one control method can be identified a priori as best. Instead, modelling should aim to identify effective interventions for a variety of release scenarios that span the ranges of uncertainty in key parameters. By attempting to identify a single 'optimal' strategy, recently published studies are arguably attempting the impossible; this is reflected in their differing conclusions, which can largely be attributed to underlying differences in model structures and parameter assignments. A more useful goal for modelling is to identify a small set of control options that might be used in a range of scenarios, together with a set of trigger thresholds, which might determine when responses need to be escalated. The key is to match model structure and aims to determine the 'necessary' level of detail.

While recognizing the limitations of modelling for precise prediction, models represent a potentially powerful resource in the face of an actual outbreak. The 2001 foot-and-mouth disease epidemic in Britain ${ }^{14-17}$ highlighted the contribution that real-time statistical analysis and modelling could make in both predicting the future course of the outbreak and identifying the measures needed for its control. For smallpox, such a role might be even more critical-to identify which of the many parameter and release scenarios explored in preparatory planning is actually being realized (for example, in relation to changes in population behaviour caused by the outbreak), and thus which set of controls is likely to be optimal in containing the outbreak while minimizing casualties. The footand-mouth story highlighted the potential advantages to be gained from having model and data structures in place as much as possible before any epidemic. In this sense recent studies ${ }^{5-8}$ and the ensuing debate are to be welcomed.

Key to such a role is how much information would be available during the early stages of an epidemic to enable the analysis to be reliable; such data would also need to give enough early warning of a 'failing' intervention to allow intensification of controls sufficiently rapidly to prevent substantial excess deaths. Investigation of this issue now, using simulation and analytical studies, is critical. Realtime data capture and dissemination is essential, and infrastructure needs to be developed in advance to facilitate this critical mechanism for implementing and monitoring interventions. Currently, it is unclear what level of detail would be necessary for models used in such a context-although some incorporation of the random effects that dominate epidemics in their early stages would clearly be desirable. If it were determined that the first few generations of cases in an outbreak would give insufficient information for realtime policy optimization, then a precautionary 'hit hard, hit early' policy might be warranted (coupled with a set of criteria for deescalating the policy), despite the higher associated costs and adverse consequences of vaccinating more people than might strictly be necessary. Such considerations are of equal relevance to the emergence of new pathogens such as $\operatorname{SARS}^{25,26}$, as they are to deliberately released agents.

doi:10.1038/nature02007.

1. Lane, H. C., Montagne, J. L. \& Fauci, A. S. Bioterrorism: a clear and present danger. Nature Med. 7, 1271-1273 (2001).

2. Dixon, C. W. Smallpox (Churchill, London, 1962).

3. Fenner, F., Henderson, D. A., Arita, I., Jezek, Z. \& Ladnyi, I. D. Smallpox and its Eradication (WHO, Geneva, 1988).

4. US sounds alarm over smallpox weapon threat. Nature 399, 628 (1999).

5. Meltzer, M. I., Damon, I., LeDuc, J. W. \& Millar, J. D. Modeling potential responses to smallpox as a bioterrorist weapon. Emerg. Inf. Dis. 7, 959-969 (2001).

6. Kaplan, E. H., Craft, D. L. \& Wein, L. M. Emergency response to a smallpox attack: The case for mass vaccination. Proc. Natl Acad. Sci. USA 99, 10935-10940 (2002).

7. Halloran, M. E., Longini, I. M., Nizam, A. \& Yang, Y. Containing bioterrorist smallpox. Science 298, 1428-1432 (2002).

8. Bozzette, S. A. et al. A model for a smallpox-vaccination policy. N. Engl. J. Med. 348, 416-425 (2003).

9. Lane, J. M. \& Goldstein, J. Evaluation of 21st-century risks of smallpox vaccination and policy options. Ann. Intern. Med. 138, 488-493 (2003).

10. Kemper, A. R., Davis, M. M. \& Freed, G. L. Expected adverse events in a mass smallpox vaccination campaign. Eff. Clin. Pract. 5, 84-90 (2002).

11. Anderson, R. M. \& May, R. M. Directly transmitted infections diseases: control by vaccination. Science 215, 1053-1060 (1982).

12. Grenfell, B. T., Bjornstad, O. N. \& Kappey, J. Travelling waves and spatial hierarchies in measles epidemics. Nature 414, 716-723 (2001).

13. Anderson, R. M., May, R. M. \& McLean, A. R. Possible demographic impact of AIDS in developing countries. Nature 332, 228-234 (1988).

14. Ferguson, N. M., Donnelly, C. A. \& Anderson, R. M. The foot-and-mouth epidemic in Great Britain: pattern of spread and impact of interventions. Science 292, 1155-1160 (2001).

15. Keeling, M. J. et al. Dynamics of the 2001 UK foot and mouth epidemic: stochastic dispersal in a heterogeneous landscape. Science 294, 813-817 (2001).

16. Keeling, M. J., Woolhouse, M. E., May, R. M., Davies, G. \& Grenfell, B. T. Modelling vaccination strategies against foot-and-mouth disease. Nature 421, 136-142 (2003).

17. Ferguson, N. M., Donnelly, C. A. \& Anderson, R. M. Transmission intensity and impact of control policies on the foot and mouth epidemic in Great Britain. Nature 413, 542-548 (2001).

18. Anderson, R. M. \& May, R. M. Infectious Diseases of Humans: Dynamics and Control (Oxford Univ. Press, Oxford, 1991).

19. Wallinga, J., Levy-Bruhl, D., Gay, N. J. \& Wachmann, C. H. Estimation of measles reproduction ratios and prospects for elimination of measles by vaccination in some Western European countries. Epidemiol. Infect. 127, 281-295 (2001).

20. Gani, R. \& Leach, S. Transmission potential of smallpox in contemporary populations. Nature 414, 748-751 (2001).

21. Eichner, M. \& Dietz, K. Transmission potential of smallpox: Estimates based on detailed data from an outbreak. Am. J. Epidemiol. 158, 110-117 (2003).

22. Bailey, N. T. J. The Mathematical Approach to Biology and Medicine (Wiley, London, 1967)

23. Koopman, J. Controlling smallpox. Science 298, 1342-1344 (2003).

24. Eichner, M. Case isolation and contact tracing can prevent the spread of smallpox. Am. J. Epidemiol. 158, 118-128 (2003).

25. Lipsitch, M. et al. Transmission dynamics and control of severe acute respiratory syndrome. Science 300, 1966-1970 (2003).

26. Riley, S. et al. Transmission dynamics of the etiological agent of SARS in Hong Kong: impact of public health interventions. Science 300, 1961-1966 (2003).

27. O’Neill, P. D. A tutorial introduction to Bayesian inference for stochastic epidemic models using Markov chain Monte Carlo methods. Math. Biosci. 180, 103-114 (2002).

28. Mollison, D. Simplifying simple epidemic models. Nature 310, 224-225 (1984).

29. Bailey, N. T. J. \& Duppenthaler, J. Sensitivity analysis in the modelling of infectious disease dynamics. Math. Biosci. 10, 113-131 (1980)

30. Isham, V. \& Medley, G. Models for Infectious Human Disease: Their Structure in Relation to Data (Cambridge Univ. Press, Cambridge, 1996)

Acknowledgements We thank the Royal Society (N.M.F. and M.J.K.), MRC (N.M.F.), Howard Hughes Medical Institute (N.M.F.), BBSRC (M.J.K. and B.T.G.), the Wellcome Trust (B.T.G. and R.M.A.) and the Department of Health (W.J.E., R.G. and S.L.) for funding. The views expressed are not necessarily those of the Department of Health, but we thank staff of the Department for discussions.

Correspondence and requests for materials should be addressed to N.M.F.

(neil.ferguson@imperial.ac.uk). 